\title{
COINCIDENCIAS Y DIVERGENCIAS ENTRE LAS PRÁCTICAS DE GESTIÓN HUMANA ADELANTADAS POR LOS DIRIGENTES-EMPRENDEDORES Y LOS DIRIGENTES-EMPLEADOS EN LAS MICROEMPRESAS DEL SECTOR HOTELERO DE LA CIUDAD DE IBAGUÉ, COLOMBIA ${ }^{1}$
}

\author{
COINCIDENCES AND DIVERGENCES BETWEEN THE HUMAN MANAGEMENT \\ PRACTICES DEVELOPED BY THE LEADERS-ENTREPRENEURS AND THE LEADERS- \\ EMPLOYEES IN THE MICRO ENTERPRISES OF THE HOTEL SECTOR IN THE CITY OF \\ IBAGUÉ, COLOMBIA
}

\author{
DOI: www.doi.org/10.54198/innova08.04 \\ Carlos Hernán Cubillos Calderón ${ }^{2}$ \\ José Vicente Montealegre González \\ Alberto Delgado Cortés ${ }^{4}$
}

Cómo citar esté artículo: Cubillos Calderón, C. H., Montealegre González, J. V., \& Cortés Delgado, A. (2021). Coincidencias y divergencias entre las prácticas de gestión humana adelantadas por los dirigentes-emprendedores y los dirigentes-empleados en las microempresas del sector hotelero de la ciudad de ibagué, Colombia, Revista Innova ITFIP, 8 (1), 33-46

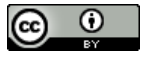

Recibido: noviembre de 2019. Aprobado: marzo de 2020

\section{Resumen}

Esta investigación se adelantó con el propósito de interpretar las prácticas de gestión humana, consideradas universales, realizadas en las microempresas del sector hotelero de la ciudad de Ibagué, pues ante la incuestionable importancia del talento humano son las grandes organizaciones y cadenas hoteleras las que cuentan con mejores posibilidades para realizar una adecuada gestión

\footnotetext{
${ }^{1}$ Esta ponencia es producto del proyecto de investigación que lleva el mismo nombre, financiado con recursos del Fondo de Investigaciones de la Universidad del Tolima y realizado por el Grupo de Investigación en Pensamiento Estratégico e Innovación Empresarial (GIPE), con el apoyo de los estudiantes del programa de Administración de Empresas María Alejandra Leal Vanegas y Edison Fabián Varón Cruz.

2 chcubi@ut.edu.co Magister en Administración. Profesor de Planta de la Facultad de Ciencias Económicas y Administrativas de la Universidad del Tolima. Investigador Grupo de Investigación en Pensamiento Estratégico e Innovación Empresarial -GIPE3 jvmontea@ut.edu.co Magister en Administración. Profesor de Planta de la Facultad de Ciencias Económicas y Administrativas de la Universidad del Tolima. Director Grupo de Investigación en Pensamiento Estratégico e Innovación Empresarial -GIPE-

4 adelgado@ut.edu.co Magíster en Administración de Empresas con especialidad en Gestión Integrada de la Calidad, Seguridad y Medio Ambiente. Profesor de Planta de la Facultad de Ciencias Económicas y Administrativas de la Universidad del Tolima. Investigador Grupo de Investigación en Pensamiento Estratégico e Innovación Empresarial -GIPE-
} 
of information collection, analysis, and interpretation were ideologically oriented by the PRODIN method (Prospective Dialectic Interpersonal Method).

Among the most relevant findings is the identification of two types of leaders: employee-leaders (non-owners) and entrepreneur-leaders (owners), who coincide in conducting informal and exploratory talks in the practice of bonding, in which intuition plays a key role in deciding on the most suitable candidate, as does the absence of standardized tests and structured or semi-structured interviews. Training, in turn, is an undervalued practice because it is assumed that the experience of the collaborators in the hotel activity is sufficient to perform efficiently. Performance evaluation is done by on-site observation of daily tasks, is not planned or systematic, and is used almost exclusively for purposes of admonishment and dismissal. The career plan as well as promotion possibilities are non-existent.

On the other hand, while the employee-leaders check their work history by phone, the entrepreneurleaders do not do this work since they privilege personal referral or recommendation. The remuneration practice also differs, since the entrepreneurs who lead their organizations generally employ family members without formally established work contracts and at the end of each month they divide the profits. In turn, the companies led by employee-leaders are far from family businesses as they do not link family members, there is a higher level of formality in the contracts and they make biweekly, monthly or shift work payments.

Keywords: Human management, managers-entrepreneurs, managers-employees, hotel sector, micro enterprises

\section{Introducción}

La gestión humana es un aspecto decisivo en el modelo de negocio de cualquier hotel, pues los servicios se materializan a través de los colaboradores quienes en buena medida trabajan en contacto directo con los clientes, de allí la importancia de contar con un equipo de trabajo adecuado que permita, de manera armoniosa, cubrir las expectativas tanto de los usuarios de estos servicios como de los mismos prestadores. Sin embargo, son las grandes organizaciones y cadenas hoteleras las que cuentan con mejores posibilidades para realizar una adecuada gestión de su talento humano en contraste con las pequeñas organizaciones que tienen recursos y capacidades limitadas, razón por la cual el Plan de Desarrollo del Departamento del Tolima (Colombia) 2016-2019 afirmara: "la transformación productiva del departamento es posible a partir de la implementación de estrategias entre las que se cuenta el fortalecimiento del talento humano" (p. 75).

Este contexto deja entrever la importancia social y económica de esta investigación, pues para contribuir al mejoramiento de las prácticas de gestión humana en las microempresas del sector 
hotelero, que para el caso de Ibagué (Colombia) corresponden al 76.32\% de los hoteles con registro nacional de turismo actualizado en el año 2018, se hace necesario caracterizarlas y comprenderlas, de tal manera que los resultados posibiliten construir líneas de acción conjuntamente con agremiaciones como la Asociación Turística y Hotelera de Colombia-COTELCO- capítulo Tolima, a fin de contribuir con el mejoramiento del personal adscrito al sector hotelero para enfrentar retos y aprovechar oportunidades.

Los hoteles pequeños son organizaciones con características especiales, por ejemplo, suelen tener un incipiente desarrollo empresarial ya que los trabajadores con baja cualificación generalmente ocupan los puestos de trabajo con mayor posibilidad de experimentar condiciones laborales inadecuadas tales como informalidad de la vinculación, prolongadas jornadas de trabajo, bajo nivel salarial, acoso laboral, discriminación, desigualdad en las oportunidades y explotación, entre otras, que impactan negativamente la prestación del servicio en una industria cuyo éxito está altamente influenciado por la constante interacción entre trabajadores y clientes. Las microempresas del sector hotelero de la ciudad de Ibagué (Colombia) no escapan a esta realidad, pues aunque desde hace algunos años se han venido planteando iniciativas sobre la base de su vocación como territorio turístico, tal como se expresara en el Plan de Desarrollo municipal 2016-2019, existe gran potencial por lograr pero asimismo mucho por hacer por lo que "se requiere un fuerte trabajo para el fortalecimiento de los prestadores y operadores de servicios turísticos locales en términos de formación y generación de capacidades para mejorar el servicio y la calidad de los servicios, atractivos y recursos turísticos (p. 122).

Ahora bien, las cadenas hoteleras que hacen presencia en la ciudad cuentan con capacidades y recursos para realizar una adecuada gestión de su talento humano, realidad contraria a la de los hoteles pequeños que representan la mayoría de establecimientos, pues según datos de la Cámara de Comercio Ibagué para el año 2018 existían, en el municipio de Ibagué, 84 hoteles urbanos con Registro Nacional de Turismo (RNT) actualizado, de los cuales 66 están catalogados como microempresas, de acuerdo con lo establecido para la época de la investigación en la Ley 905 de 2004, pues ocupan hasta 10 trabajadores en la prestación de sus servicios. A lo anterior, se suma el hecho que las prácticas de gestión humana en las microempresas y particularmente en las que se dedican al hospedaje y alojamiento han sido poco estudiadas, prueba de ello es el bajo número de resultados investigativos reportados en bases de datos y plataformas académicas sobre este tema, salvo algunos trabajos de tesis que no han aportado significativamente a la construcción de teoría sobre el particular que oriente la gestión humana en este tipo de organizaciones. 
REVISTA INNOVA ITFIP, 8 (1). 33 - 46. JUN. 2021

\section{Objetivo General}

Caracterizar e interpretar las prácticas de gestión humana en las microempresas del sector hotelero de la ciudad de Ibagué.

\section{Objetivos Específicos}

- Estudiar las prácticas de vinculación de personal en los pequeños hoteles de la ciudad de Ibagué.

- Analizar las prácticas de capacitación del personal que se realizan en los pequeños hoteles de Ibagué.

- Examinar las prácticas de compensación del personal en los pequeños hoteles de la ciudad de Ibagué.

- Analizar las prácticas de evaluación del desempeño en los pequeños hoteles de la ciudad de Ibagué.

- Interpretar las prácticas de permanencia y proyección del personal en los pequeños hoteles de la ciudad de Ibagué.

\section{Materiales y Métodos}

Este proyecto de investigación se desarrolló bajo el enfoque cualitativo, orientado a partir de los planteamientos básicos de la Grounded Theory (Teoría fundada o teoría fundamentada) - TF planteada por Glaser y Strauss (1967) como método interpretativo de la realidad social. Las fases de recopilación, análisis e interpretación de la información fueron orientadas ideológicamente por el método PRODIN (Prospective Dialectic Interpersonal Method), propuesto y desarrollado por Bergadaá (2006).

Determinación de los sujetos de estudio. Según datos de Cámara de Comercio de Ibagué (Colombia), para el año 2018 existen 84 hoteles urbanos con Registro Nacional de Turismo (RNT) actualizado, de los cuales 66 se clasifican como microempresas. La escogencia de los once (11) sujetos de estudio se realizó de forma progresiva en la medida que se presentaron hallazgos que aportaron nuevos elementos y cuando los datos se repitieron no se entrevistaron nuevos sujetos puesto que la investigación entró en la etapa de saturación teórica.

Recolección y análisis de la información. Como fuente de información primaria se consultaron, a través de entrevistas semiestructuradas, los pequeños hoteleros de la ciudad de Ibagué. Se realizaron y trascribieron las primeras entrevistas sobre las cuales se hizo un "análisis piloto". El nuevo formato guía de entrevista se aplicó a los otros entrevistados, generando audios que fueron trascritos literalmente conservando su fidedignidad y posteriormente se realizó el análisis microscópico de los datos (frase por frase) Esta información depurada permitió estructurar las categorías descriptivas. 
Validez y fiabilidad. Los análisis de contenido fueron sometidos a pruebas de validez interna (triangulación de investigadores) entendida como el grado en el que se pueden establecer relaciones causales (Yin, 1989) y validez de constructo, con el propósito de evitar juicios subjetivos por parte del equipo investigador en la recolección de datos a través de la revisión de los resultados por parte de expertos.

\section{Marco teórico}

Desde hace varias décadas la literatura y el medio profesional sitúan los activos intangibles como principales factores críticos del éxito organizacional y conceden a las personas el papel protagónico como fuente de ventaja competitiva, orientación ratificada en los trabajos de Camisón (1997), Capelli (1999), Hitt, Bierman, Shimizu y Kochhar (2001), DeNisi, Hitt y Jackson (2003) además de Becker, Huselid y Beatty (2009). A pesar de este consenso, investigadores como Storey (1992), Rowden (1995), Wagar (1998) Calderón y Álvarez (2006), Calderón, Naranjo y Álvarez (2010), advierten la relativa escases en el número de investigaciones relacionadas con las prácticas de gestión humana en las pequeñas y medianas empresas, y reconocen que no necesariamente las prácticas implementadas en las grandes empresas son las apropiadas para las pequeñas.

De otra parte, Cassell y colaboradores (2002) citados en Torres (2014, p. 40) señalan la existencia de cuatro prácticas consideradas universales en gestión humana, pues independientemente de cómo se realicen, quién y con qué mecanismos las apliquen, todas las organizaciones -incluidas las pequeñas realizan procesos de selección, evaluación de desempeño, remuneración y desarrollo, mientras que Paauwe y Boselie (2008) identifican 26 prácticas, de las cuales privilegian las cuatro primeras: entrenamiento y desarrollo, esquemas de retribución, gestión de desempeño, y reclutamiento y selección.

A su vez, Camarena y Saavedra (2018) destacan el reclutamiento, selección, capacitación, inducción y desarrollo, mientras que la firma Interconsulting Bureau, propone como funciones básicas "las actividades, métodos y medios para reclutar y seleccionar personal, formarlo, integrarlo, promocionarlo o degradarlo, transferirlo o suspenderlo y otras actividades que afectan a la motivación del personal y al clima sano y estimulante de la estructura de la empresa" (2015, p. 42).

Allens (2006), Dessler (2004) y Dolan (2002) citados en Rodríguez (2012) coinciden en la determinación de las principales prácticas de gestión humana: descripción y análisis de cargos, vinculación, formación y entrenamiento, remuneración y beneficios, evaluación del desempeño y plan de carrera, planteamiento muy similar a las denominadas prácticas genéricas en gestión humana por los investigadores colombianos Calderón y Álvarez (2006), Calderón, Naranjo y Álvarez (2007) y Fonseca (2013): vincular trabajadores (práctica de provisión de personal), entrenarlos y formarlos (práctica de capacitación), remunerarlos (práctica de compensación), 
supervisarlos y orientarlos (práctica de evaluación de desempeño) y tener algún criterio de permanencia y proyección en la empresa (práctica de plan de carrera), planteamiento que se acoge como orientación teórica del presente proyecto de investigación.

De acuerdo con lo anterior, "la provisión comprende tres prácticas diferenciadas - el reclutamiento, la selección y la contratación -" (Valle 2004 citado en Calderón y Álvarez 2006 p. 32). A su vez, Chiavenato (2017) plantea: "la capacitación es el proceso educativo de corto plazo, aplicado de manera sistemática y organizada, por medio del cual las personas adquieren conocimientos, desarrollan habilidades y competencias" (p. 330). Como compensación, Dolan, Valle-Cabrera, Jackson \& Schuler (2003) consideran el conjunto de precepciones financieras, servicios o beneficios tangibles que recibe un empleado como consecuencia de la prestación de la actividad a la empresa, en la que se reconoce la retribución directa normalmente de carácter monetario y la retribución indirecta que corresponde a los beneficios que recibe el trabajador. A su vez, Torres (2014) en relación con la evaluación del desempeño en pequeñas organizaciones plantea: "se utiliza como medio de control con fines administrativos; es decir como insumo de información para despidos, aumentos salariales, bonificaciones, reconocimientos y recompensas no monetarias como horarios flexibles" (p. 24). Por otra parte, Werther \& Davis afirman: "cuando la administración de la empresa alienta la planeación de la carrera, es más probable que los empleados se fijen metas profesionales y que trabajen activamente por obtenerlas" (2000, p. 273).

\section{Resultados y Discusión}

\section{Tipología de los dirigentes de las microempresas del sector hotelero.}

Entre los resultados más relevantes se destaca una tipología de los directivos compuesta por dos grandes categorías: los dirigentes-empleados y los dirigentes-emprendedores, en la primera se identifican dirigentes empíricos y otros con formación profesional. La segunda se divide entre los que cuentan con experiencia previa en el sector y aquellos emprendedores que no se dedicaban a la actividad hotelera.

\section{Dirigentes-empleados}

En esta categoría se ubican los dirigentes que no cuentan con formación profesional en negocios, pero que tienen alguna experiencia laboral previa en otras actividades productivas relacionadas, principalmente, con el sector agrícola, el comercio detallista y las manufacturas, así como quienes estaban desempleados y encontraron en este sector una ubicación laboral, tal como lo manifiesta el informante No. 7 "yo estaba buscando empleo y se me presentó esta oportunidad, me hicieron una entrevista y hasta el momento pues estoy acá...". Se ocupan de labores básicas de administración relacionadas con la preparación de información contable, control del inventario de insumos, supervisión del mantenimiento y aseo del hotel, acciones que han aprendido a realizar de manera empírica: "uno va aprendiendo por el camino...de las cosas que se van presentando..." (Informante No. 3). En términos generales, poseen varios años vinculados a la misma organización, 
REVISTA INNOVA ITFIP, 8 (1). 33 - 46. JUN. 2021

incluso algunos desde la fecha de fundación, no cuentan con parientes en la misma línea de negocio y los trabajadores que emplean no son miembros de su familia.

Asimismo, son representantes de esta categoría los profesionales que se ubicaron laboralmente como administradores. Iniciaron su experiencia laboral en actividades económicas diferentes a la hotelería, pero se fueron relacionando con el medio pues sus empleadores vieron en ellos capacidades, aptitudes, habilidades y formación académica para dirigir sus negocios desde una perspectiva profesional. Buena parte de su tiempo la ocupan en las funciones de supervisión y control de las actividades desarrolladas por sus colaboradores; así, como a procesos relacionados con el mejoramiento en la atención de los huéspedes tales como solución de peticiones, quejas y reclamos. Valoran la formación permanente ofrecida por agremiaciones y entidades del sector: "desde que abrimos el hotel nos vinculamos a COTELCO...tienen un programa de capacitación muy bueno...entonces...y yo he accedido a todo ese tipo de capacitaciones para poder conocer desde cómo tender a una cama hasta cómo hacer un estado financiero” (Informante No. 9).

Buena parte de los dirigentes de esta categoría trabajaron inicialmente como empleados en el sector hotelero en diversos cargos y decidieron emprender un proyecto empresarial apoyándose en la experiencia laboral que poseían, como es el caso del informante No. 1: "siempre he manejado lo que era mantenimiento, ya ahorita como soy directamente el arrendatario, estoy respondiendo por esto, entonces me enfoque ya en lo que es la parte administrativa". A su vez, el informante No. 5 afirma que la decisión de convertirse en empresario fue motivada por su experiencia laboral, "era administrador de una empresa hotelera y entonces decidi tener mi propio negocio”. Son dirigentes polifuncionales; en un día normal pasan de hacer el aseo de las instalaciones y atender a los clientes en la recepción, a hacer cuadres de caja, compras y llevar la contabilidad del negocio. No cuentan con formación profesional, pero son el centro del negocio pues ejercen el poder, el control y la toma de decisiones con alta autonomía.

Algunos de ellos se iniciaron en esta actividad económica a partir de sucesos detonantes negativos como la pérdida del empleo o el desplazamiento del campo a la ciudad, tal como relata el informante No. 4: "estaba tocando puertas... ¿dónde hay un trabajo? ¿quién me recibe? entonces había un letrerito: se arrienda hospedaje, entonces yo decidí tomar, dije, me voy a montar en este caballo loco". Estos dirigentes emplean a sus familiares y alternan las funciones laborales (administrativas y operativas) con su rol familiar al compartir espacios cotidianos y celebraciones especiales en las instalaciones del hotel, debido a que su jornada laboral fluctúa entre las ocho y las 16 horas diarias.

\section{Prácticas genéricas de gestión humana en las microempresas del sector hotelero.}

\section{Vinculación del personal}

En las microempresas del sector hotelero se presenta muy poca rotación de personal por lo que son muy esporádicos los procesos de vinculación. Sin embargo, la necesidad de emplearse hace que muchas personas de manera espontánea dejen a consideración de los hoteles sus currículos (hojas 
de vida): "viene mucha gente buscando empleo y...pues ahorita que estamos con ese recorrer de venezolanos, eso es mucha la gente que viene y le trae a uno la hoja de vida, y son perfiles muy buenos" (informante No. 8), o como tal como lo expresa la informante No. 9: "sí...vienen o nos escriben al correo, nos lo mandan por Facebook, que conozco el área, soy estudiante de hotelería, o trabaja en un hotel y nos mandan las hojas de vida". La iniciativa de dejar las hojas de vida directamente en las empresas, así como la recomendación personal se constituyen en las dos fuentes de reclutamiento del personal con mayor reconocimiento.

Por su parte, los dirigentes-empleados que han adelantado procesos de vinculación de personal recurren a la verificación telefónica de antecedentes laborales, la realización de entrevistas desestructuradas y a la percepción que logran sobre el candidato para que este último continúe en el proceso con una corta inducción que busca valorar la competencia del candidato frente a las actividades y responsabilidades propias del empleo: “...se convoca a entrevista, se mira el perfil, se tiene la charla con la persona, ¿sí? uno mira que le vea aptitudes, que encaja, pues entonces ya procede a decirle que venga a un proceso de inducción" (Informante No. 8). Los pequeños hoteles liderados por dirigentes-empleados cuentan con una sencilla pero definida estructura organizacional en la que se reconocen dos niveles: el administrativo que corresponde al cargo ocupado por el dirigente y el operativo al que se asimilan los cargos de recepcionista, camarero y oficios varios.

En contraste a lo anterior y aunque existe considerable literatura en la que se describen puntualmente métodos para realizar el reclutamiento y la selección del personal, los dirigentesemprendedores que lideran microempresas del sector hotelero privilegian, para efectos de vinculación del personal, la recomendación personal o referenciación, es decir, la sugerencia que hacen familiares, conocidos o empleados sobre aquellas personas que consideran como buenos trabajadores, lo que se constituye en una garantía, para el dirigente, que el aspirante al cargo será el trabajador que se requiere. Estos dirigentes no revisan antecedentes laborales pues confían en la recomendación que recibieron, tampoco realizan pruebas estandarizadas de orden técnico o psicológico, no efectúan entrevistas estructuradas, sino que realizan charlas informales, exploratorias, desestructuradas con los aspirantes a trabajadores en las que la intuición juega un papel importante para determinar el candidato más idóneo.

La contratación a su vez no está mediada por un documento escrito ni por las formalidades que son comunes a otras organizaciones, sino que obedece a un acuerdo verbal entre las partes en las que el dirigente impone las condiciones. Asimismo, como buena parte de los empleados son familiares cuando se presentan ausencias de algún trabajador otro lo reemplaza cubriendo su turno para el normal funcionamiento de su negocio: "cuando viaja la hija, pues entonces el hijo es el que los reemplaza...nosotros no necesitamos de contrato, nos repartimos lo que queda” (Informante No. 2). 


\section{Capacitación}

Para los microempresarios del sector hotelero la capacitación se entiende, casi de manera exclusiva, como inducción, es decir orientaciones básicas que el empleado recibe durante la vinculación: "[la capacitación] puede durar ocho días, dos días, eso sí depende de la capacidad que tenga la persona para absorber lo que se le diga" (Informante No. 6). Cuando se realiza en otro momento de la relación laboral, generalmente es desarrollada por el mismo empresario y comprende instrucciones básicas e informales que se imparten durante la ejecución de las actividades, pues como afirma la informante No. 2: "trabajando sobre terreno es que uno aprende...". No obstante, en algunos hoteles liderados por dirigentes-empleados se hace uso frecuente de capacitaciones gratuitas impartidas por agremiaciones del sector en temas que se enfocan a la calidad del servicio o a actividades operativas propias del negocio.

La capacitación no corresponde con un plan previamente concebido, con una intencionalidad clara, con un mecanismo que permita validar los resultados en relación con el mejoramiento de los procesos. El empirismo con que se administra el negocio hace que el empresario asuma que el trabajador, por su experiencia en el cargo, posee todos los conocimientos, destrezas y aptitudes que le permiten desempeñarse de manera eficiente, lo que a su vez redunda en la subvaloración de esta práctica de gestión humana: "no... acá no se maneja eso, es prácticamente porque como... las personas que están acá ya han trabajado en hotel" (Informante No. 10). La capacitación incluso es vista como un gasto y no como una inversión, pues en términos de uno de los dirigentesemprendedores: "no gustamos de la academia, o del horario... porque... muchas veces son hasta gratis... se trata de... dedicarle tiempo, y como a uno no le pagan como que parece que eso es cansón..." (Informante No. 11), o porque simplemente se considera irrelevante: "con lo que se hace acá diario, yo creo que esa es la capacitación" (Informante No. 7).

\section{Compensación}

En las microempresas del sector hotelero no existe unidad de criterio en cuanto a la remuneración, pues en aquellas en las que el dirigente es empleado y no ocupan familiares, generalmente se paga por día laborado mientras que otras reconocen el salario mínimo mensual legal vigente para cargos como el de camarero y recepcionista, y efectúan los pagos correspondientes al salario básico, el auxilio de transporte y los reconocimientos por horas extras, recargos nocturnos, dominicales y festivos, cada quince días. Sin embargo, la unidad de trabajo más común es el turno que implica el pago entre 9 y 15 dólares americanos por una jornada laboral que se establece comúnmente entre ocho y doce horas, pero puede llegar hasta las 24 horas. Los dirigentes consideran que el pago que reciben sus empleados está dentro del promedio salarial del sector productivo, en palabras de la informante 4 "yo no diría que [pago] mejor [que los demás], pero si está dentro del rango..." y, del informante 10: "...yo me imagino que todos pagaran lo mismo", por lo que se infiere que desconocen el nivel salarial de la competencia. 
En términos generales, en las organizaciones que cuentan con empleados familiares del emprendedor no se tienen contratos de trabajo formalmente establecidos "no tenemos contrato de trabajo porque nosotros acá trabajamos por un sueldo por un poco más del mínimo primero que todo, y segundo como es un negocio familiar a nadie le vamos a cobrar ni prestaciones, ni nada de eso" (Informante No. 6), tampoco se pagan salarios fijos, recargos, prestaciones o auxilio de transporte, debido a que se reciben otros beneficios diarios en especie como alimentación, alojamiento y vestido, en algunas ocasiones. Además, el monto y la periodicidad de la retribución se realiza de acuerdo con la disponibilidad del efectivo y a las necesidades de la familia, pues según el informante No. 11: "pues si toca [pagar]...quincenal, o toca diario...como se necesite”.

Asimismo, en cuanto a los pagos en dinero, el dirigente-emprendedor divide las utilidades entre los empleados-familiares al finalizar el mes, una vez se descuentan los pagos realizados en especie y los costos y gastos de funcionamiento del hotel: "nosotros no necesitamos de contrato, nos repartimos lo que queda... [el pago] es según lo queda del mes, asimismo toca... si quedaron quinientos pues, quinientos, si quedaron seiscientos, seiscientos, si nos quedaron cuatrocientos, cuatrocientos" (Informante No. 2).

\section{Evaluación del desempeño}

La evaluación del desempeño como herramienta que contribuye con el mejoramiento organizacional y el logro de los objetivos empresariales no reviste ningún procedimiento formal, no es programada, ni sistemática sino que es realizada por el dirigente a través de la observación directa de las labores realizadas por el trabajador, obedece fundamentalmente a la supervisión directa de las actividades programadas de manera diaria o semanal, situación que puede verificarse en afirmaciones como: "el control es que... entre más ligero haga usted el oficio y déjelo bien hecho, y ya..." (Informante No. 2), o tal como asegura el informante No. 1: "la supervisión se realiza directamente saliendo a campo.... y cuando uno no está, por medio de las cámaras".

Se recurre a la corrección de las acciones en el momento mismo de su realización a través de orientaciones o amonestaciones verbales, no se lleva registro alguno de los hechos que merecen ser corregidos, así como tampoco de las acciones sobresalientes que ameritan reconocimiento. El eventual despido de un trabajador puede originarse en conductas que van en contra de las actuaciones que desea el empleador de sus empleados así sean familiares: "la familia se queda afuera en la puerta... allá hay un... árbol genealógico en la puerta, usted me deja toda su familia ahi y me entra solita, ni yo soy su familiar" (Informante No. 4), con lo que se puede inferir que la evaluación del desempeño de los empleados-familiares se hace de manera similar a la evaluación de los trabajadores-no familiares puesto que el hotel debe funcionar como cualquier organización y que las actividades se deben efectuar como el dirigente indique. Se asume la comunicación entre el dirigente y sus empleados-familiares como aspecto clave del control: "...lo importante es la comunicación y, como trabaja la familia y, además, los que trabajamos acá tenemos la experiencia, entonces tampoco se briega mucho..." (Informante No. 6). 


\section{Prácticas de permanencia y proyección del personal}

En lo concerniente a las prácticas de permanencia, las microempresas objeto de estudio no cuentan con políticas o planes formales sobre el particular y, las acciones que se ejecutan día a día no están dirigidas de manera deliberada a incentivar la permanencia de los colaboradores, sino que se orientan a partir de percepciones personales sobre la relación entre el dirigente, los trabajadores y los clientes. Algunas de las acciones inciden en el clima organizacional, aspecto que tiene efecto sobre la permanencia del personal, pues tal como manifiesta uno de los dirigentes: “... lo que más se comparte en esta familia es... como los cumpleaños; ;siempre hay un ponqué... hay una CocaCola! iSiempre hay un pedacito para el que no estuvo!” (Informante No. 11). Adicionalmente, otros aspectos que inciden en la permanencia de los colaboradores se asocian con la edad y la cualificación laboral: "ellos saben que... a estas alturas de la vida, quien les va pagar lo mismo" (Informante No. 3).

El plan de carrera en estas organizaciones es inexistente, así como las posibilidades de ascenso y las perspectivas de crecimiento, lo que se justifica desde aspectos como el bajo número de cargos y las limitaciones de la estructura organizacional: “...quien llega a la recepción... llega a la recepción, quien llega a la parte del aseo... llega a esa parte, y listo y ya...” (Informante No. 6). De acuerdo con lo revelado por el informante No. 3: en los 19 años que lleva dirigiendo su hotel no se han presentado casos de ascensos laborales, sin embargo, aclara que en su empresa algunos empleados temporalmente pueden ocupar otros cargos: “...pongamos los domingos, en la recepción no hay sino hombres" razón por la cual argumenta que todos sus empleados deben conocer las funciones de otros puestos de trabajo, en especial de la recepción para cubrir los turnos dominicales y festivos, sin embargo, esta flexibilidad no implica un ascenso laboral.

\section{Conclusiones}

Las principales coincidencias identificadas en las prácticas de gestión humana que adelantan los dirigentes-emprendedores y los dirigentes-empleados (no propietarios) en las microempresas del sector hotelero tienen que ver con la realización de charlas informales y exploratorias en las que la intuición juega un papel fundamental para decidir sobre el candidato más idóneo. Asimismo, están ausentes las pruebas estandarizadas de orden técnico o psicológico, así como las entrevistas estructuradas o semiestructuradas durante la selección de personal. En la práctica de capacitación, los sujetos de estudio concuerdan al asumir que los candidatos con experiencia laboral específica cuentan con los conocimientos, destrezas y aptitudes que les permitirán desempeñarse de manera eficiente en el cargo, lo que redunda en la subvaloración de esta práctica de gestión humana.

Igualmente, se identificó sincronía en la evaluación del desempeño pues esta práctica no reviste formalismo alguno, no es programada y sistemática, sino que es realizada por los dirigentes (emprendedores y empleados) mediante de la observación directa de las labores diarias realizadas 
por el trabajador, pero no se llevan registros de reconocimientos o de llamados de atención. Se emplea fundamentalmente con fines de amonestación verbal y eventuales despidos. Asimismo, se encontraron similitudes en la práctica de permanencia y proyección de personal, toda vez que no se identificaron políticas o planes formales sobre el particular. Aunque el clima organizacional se considera importante, las acciones diarias no se dirigen a incentivar la permanencia de los trabajadores, sino que esta última está influenciada en buena medida por la inexistencia de cualificación laboral para desempeñarse en otras actividades productivas. El plan de carrera en las organizaciones estudiadas es inexistente al igual que las posibilidades de ascenso.

Entre las divergencias más significativas se pueden mencionar aquellas que corresponden al proceso de vinculación del personal, pues mientras los dirigentes-empleados verifican telefónicamente los antecedentes laborales, los dirigentes-emprendedores no realizan esta labor dado que privilegian la recomendación personal o referenciación, es decir, la sugerencia que hacen familiares, conocidos o empleados sobre aquellas personas que consideran buenos trabajadores, lo que se constituye en una garantía, para el dirigente. Tampoco realizan pruebas estandarizadas de orden técnico o psicológico, no efectúan entrevistas estructuradas sino charlas informales y exploratorias en las que la intuición juega un papel fundamental para decidir sobre el candidato más idóneo.

La práctica de remuneración igualmente presenta diferencias, pues los emprendedores que lideran sus organizaciones generalmente ocupan familiares sin contratos de trabajo formalmente establecidos y al finalizar cada mes dividen las utilidades generadas con los empleados-familiares una vez se han descontado los pagos realizados es especie, así como los costos y gastos en que se incurre para el funcionamiento del hotel. A su vez, las empresas lideradas por dirigentes-empleados distan de las famiempresas en tanto no se vinculan familiares, hay mayor nivel de formalidad en los contratos de trabajo que en las organizaciones lideradas por emprendedores, se efectúan pagos quincenales o mensuales de acuerdo con lo previsto en la Ley, pero la unidad de trabajo más común es el turno que corresponde a una jornada que generalmente va entre 8 y 12 horas.

\section{Referencias}

Alcaldía de Ibagué. (2016). Plan de Desarrollo Municipal 2016-2019-Por Ibagué con todo el corazón. Ibagué, Colombia

Asociación Turística y Hotelera de Colombia -COTELO- (2012). Estudio básico laboral de hoteles afiliados a COTELCO

Calderón, G., Naranjo, J. C. \& Álvarez C. M. (2010). La empresa colombiana: sus características, retos y aportes. Una aproximación a un sistema integral. En revista Cuadernos de Administración, Universidad Javeriana, volumen 23 No. 41, p 13-36. Bogotá, Colombia

Calderón, G., Naranjo, J. C. \& Álvarez C. M. (2007). La gestión humana en Colombia: características y tendencias de la práctica y de la investigación. En revista Estudios Gerenciales, Universidad ICESI, volumen 23 No. 103, p 13-36. Bogotá, Colombia 
REVISTA INNOVA ITFIP, 8 (1). 33 - 46. JUN. 2021

Calderón, G. \& Álvarez C. M. (2006). Características y sentido de las prácticas de gestión humana en pequeñas empresas. En revista Universidad EAFIT. Volumen 42 No. 142, p 26-45. Medellín, Colombia

Cámara de Comercio de Ibagué (2018). Caracterización perfil empresarial del sector turismo (2018) y su dinámica (2017-2018)

Camarena, M. E. \& Saavedra, M. L. (2018) Las prácticas de capital humano en las Pymes de la Ciudad de México Forum Empresarial, vol. 23, núm. 1, Universidad de Puerto Rico, Puerto Rico Disponible en: http://www.redalyc.org/articulo.oa?id=63157547008

Chiavenato, I. (2017). Administración de recursos humanos. Editorial Mc Graw Hill. México Centro de Estudios de Opinión-CEO- (sf). Teoría fundada: arte o ciencia. Universidad de Antioquia, Facultad de Ciencias Sociales y Humanas, Medellín, Colombia.https://aprendeenlinea.udea.edu.co/revistas/index.php/ceo/article/viewFil e/1632/1285

Congreso de Colombia (2004) Ley 905, por medio de la cual se modifica la Ley 590 de 2000 sobre promoción del desarrollo de la micro, pequeña y mediana empresa colombiana

Dolan, S. L., Valle-Cabrera, R., Jackson S. E. \& Schuler, R. S. (2003). La gestión de los recursos humanos; Cómo atraer, retener y desarrollar con éxito el capital humano en tiempos de transformación. Tercera edición, Editorial Mc Graw Hill

Fonseca, P. (2013). Prácticas de gestión humana en empresas del sector comercio de la ciudad de Tunja dedicadas al comercio de muebles y electrodomésticos. En Revista Ensayos, año 6 No. 6, p 127139. Manizales, Colombia

Glaser, B. \& Strauss, A. (1967). The discovery of grounded theory. Chicago: Aldine Press.

Interconsulting Bureau S. L. (2015). Planificación y gestión de recursos humanos. ICB Editores. Málaga, España

Paauwe, J. \& Boselie, P. (2005). HRM y rendimiento: ¿Qué sigue? (Documento de trabajo CAHRS \# 0509). Ithaca, NY: Universidad de Cornell, Escuela de Relaciones Industriales y Laborales, Centro de Estudios Avanzados de Recursos Humanos.http://digitalcommons.ilr.cornell.edu/cahrswp/476

Rodríguez, D. C. (2012). Prácticas de gestión humana en pequeñas empresas. En revista Apuntes del CENES. Volumen 31 No. 54. Universidad Pedagógica y Tecnológica de Colombia, Tunja, Colombia, p 193-226

Torres, C. C. (2014). Tarea pendiente: la gestión de recursos humanos en las PYMES. En Revista Debates IESA, Volumen XIX No. 4.

Werther, W. B. Davis, K. (2000). Administración de personal y recursos humanos. Editorial Mc Graw Hill. Quinta edición. México Yin, R. (1994). Case Estudy Research. Desing and Methods. Sage publications, Thousand Oaks, CA 